Next, we analyzed signaling molecules activated in CD11b cells pulsed with PD-L1 \pm CD200AR-L, followed up with in vitro and in vivo effects of CD200AR-L on the expression of PD-1/PD-L1 and CTLA-4. Finally, we analyzed the ability of the CD200AR-L to surmount the suppressive effects of PDL1.

Results Our studies demonstrate that the inhibitory CD200R1 and PD-1 mediate immune checkpoint signaling activities through SHIP1 protein. Moreover, CD200AR-L overpowers the suppressive effects of CD200 and PD-L1, which are both shed by tumors, by downregulating the inhibitory CD200R1 and PD-1 on both antigen-presenting cells (APC) and T-cells (figure 1), In addition, CD200AR-L downregulates PD-1 on APCs and inhibits the upregulation of PD-L1 and CTLA4.

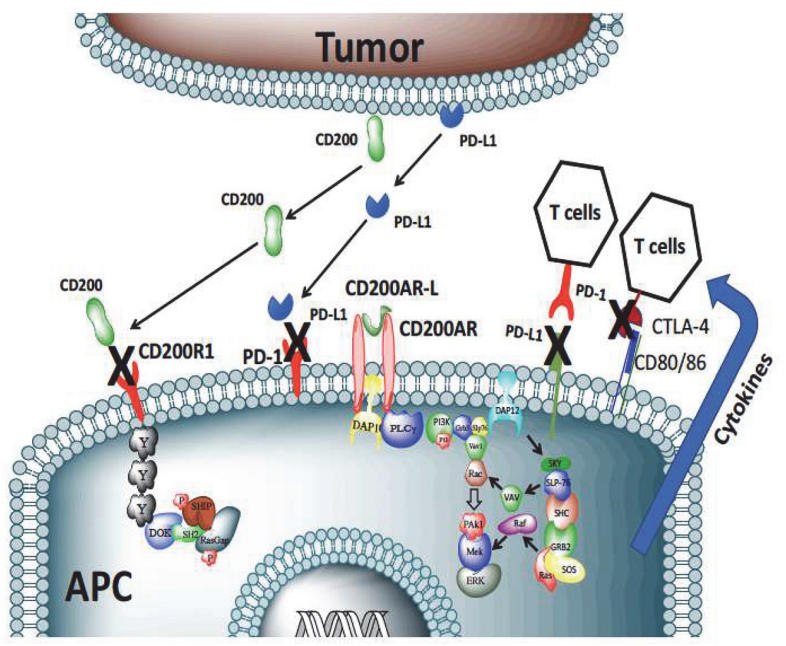

Abstract 213 Figure 1 Mechanism of the CD200 Checkpoint Ligand

Conclusions These studies led to the discovery that this novel peptide modulates the CD200, PD-1/PD-L1 and CTLA-4 pathways, providing the basis for the translatable development of a novel CD200 peptide inhibitor for clinical use against multiple tumors, including gliomas. These studies led to the FDA approval for the first in human peptide checkpoint inhibitor to initiate a phase I single center, open-label, dose-escalation clinical trial in adult patients with recurrent glioblastoma, to be followed by a clinical trial for children with recurrent malignant brain tumors.

http://dx.doi.org/10.1136/jitc-2020-SITC2020.0213

\section{THE EFFECT OF ANTI-PD-1 THERAPY ON MEDIAN OVERALL SURVIVAL AND PROGRESSION FREE SURVIVAL IN GLIOBLASTOMA MULTIFORME PATIENTS WITH CERTAIN TUMOR MARKERS}

Awais Paracha*, ' Jian Campian. 'Saint Louis University Medical School, Saint Louis, MO, USA; ${ }^{2}$ Washington University, Saint Louis, MO, USA

Background Almost 1 in 6 malignant brain cancers are Glioblastoma Multiforme, relative to most other brain cancers it is the most aggressive and prevalent by the numbers. ${ }^{1}$ Even with the best treatment options median Overall Survival(OS) remains morbid at 14.6 months and Progression Free Survival (PFS) remains 6.9 months. ${ }^{2}$ Telomerase Reverse Transcriptase promoter mutations, ${ }^{3}$ Isocitrate Dehydrogenase(IDH) mutations, ${ }^{4}$ and Tumor Mutation Burden $(\mathrm{TMB})^{5}$ are three prominent tumor markers that are known to be associated with better PFS and OS; markers like these in the presence of new therapies maybe prove crucial to the development of novel therapies. Immunotherapy has been dubbed a 'game changer' in certain hematological and solid malignancies. Specifically, PD1 is a glycoprotein that is a strong negative regulator of the immune system, by blocking this glycoprotein AntiPD-1 agents harness a strong response by the immune system to fight a malignancy ${ }^{6}$. In conjunction with these new found tumor markers, Anti-PD-1 agents maybe the solution that could dramatically improve OS and PFS in these patients.

Methods The goal of this study was to retrospectively analyze patients' charts who had received Anti-PD-1 therapy and had TERT promoter mutations, IDH mutations, different TMBs, and other markers and to compare their OS and PFS outcomes with conventional therapies and their response to immunotherapy.

Results Upon analyzing the data the presence of a TERT promoter $124 \mathrm{C}>\mathrm{T}$ mutation, IDH wildtype, and lower TMB gave much better OS and PFS after treatment in patients on Anti-PD1 therapy.

Conclusions Although this was a small study, these results certainly can be used to examine larger subsets of patients with these markers receiving immunotherapy because they had definitively better outcomes as compared to status quo treatment options.

Ethics Approval The study was approved by Washington University Ethics Board, approval number 201111001.

\section{REFERENCES}

1. Davis, M.E., Glioblastoma: overview of disease and treatment. Clinical journal of oncology nursing, 2016;20(5 Suppl): p. S2-S8.

2. Stupp $\mathrm{R}$, et al., Radiotherapy plus concomitant and adjuvant temozolomide for glioblastoma. New England Journal of Medicine 2005;352(10): p. 987-996.

3. Mosrati MA, et al., TERT promoter mutations and polymorphisms as prognostic factors in primary glioblastoma. Oncotarget 2015;6(18): p. 16663-16673.

4. Chen J-R., et al., Isocitrate Dehydrogenase (IDH) $1 / 2$ Mutations as Prognostic Markers in Patients With Glioblastomas. Medicine, 2016;95(9): p. e2583-e2583.

5. Wu $Y$, et al. The predictive value of tumor mutation burden on efficacy of immune checkpoint inhibitors in cancers: a systematic review and meta-analysis. Frontiers in Oncology 2019;9:p. 1161-1161.

6. Almåsbak H, Aarvak T, and Vemuri MC, CAR T. Cell Therapy: a game changer in cancer treatment. Journal of Immunology Research 2016;2016:p. 54746025474602.

http://dx.doi.org/10.1136/jitc-2020-SITC2020.0214

\section{A0-176, A HIGHLY DIFFERENTIATED CLINICAL STAGE ANTI-CD47 ANTIBODY, PREFERENTIALLY BINDS TUMOR VERSUS NORMAL CELL CD47 WHEN COMPLEXED TO $\beta 1$ INTEGRIN}

Robyn Puro*, Prabir Chakraborty, John Richards, Ronald Hiebsch, Michael Donio, W Casey Wilson, Benjamin Capoccia, Carrie Brachmann, Vicki Sung, Arun Kashyap, Daniel Pereira. Arch Oncology, St. Louis, MO, USA

Background Overexpression of CD47 by tumor cells exploits an immune checkpoint preventing tumor recognition and destruction by innate immune cells. Binding of tumor CD47 to SIRP $\alpha$ on macrophages and dendritic cells triggers a 'don't eat me' signal that inhibits phagocytosis and allows escape from innate immune surveillance. Blockade of the CD47/ SIRP $\alpha$ axis, however, enables immune recognition and phagocytic clearance of tumor cells. We have developed a clinical stage CD47 targeting antibody AO-176 that is highly differentiated among agents in this class. AO-176 not only blocks the 
CD47/SIRP $\alpha$ interaction and induces phagocytosis of tumor cells, but it also has a direct killing mechanism (via PCDIII) and induction of immunogenic cell death, leveraged by preferential binding to tumor versus normal cell CD47.

Methods CD47 and $\beta 1$ integrin expression and localization were evaluated using a combination of flow cytometry, western blotting, confocal microscopy and immunohistochemistry.

Results Previously, we described that the preferential binding of AO-176 to tumor versus normal cells was due to its interaction with CD47 molecules that were pre-complexed to $\beta 1$ integrin. This finding was particularly important and suggestive of why AO-176 does not bind red blood cells since they do not express $\beta 1$ integrin. We have extended these findings to show that $\beta 1$ integrin as well as CD47 are also expressed at lower levels in normal versus tumor cells, and that solid and hematologic tumor cells overexpress both CD47 and $\beta 1$ integrin which correlate with poor prognosis in cancer. In addition, we show that AO-176 is able to bind and occupy CD47/ 31 integrin complexes to a greater extent at acidic versus physiologic $\mathrm{pH}$ such as would be found in tumor microenvironments, an observation that also contributes to the enhanced targeting of AO-176 to tumor cells. Taken together, these findings add further insight into the preferential binding of AO-176 to tumor versus normal cells.

Conclusions The context dependent binding of AO-176 to CD47, when complexed to $\beta 1$ integrin, is unique among CD47 axis targeting agents and together with its direct killing mechanism of action offers a potentially better safety profile and opportunity for a therapeutic advantage. AO-176 is currently being evaluated in Phase 1 clinical trials for the treatment of patients with select solid tumors (NCT03834948) and multiple myeloma (NCT04445701).

Trial Registration NCT03834948, NCT04445701.

http://dx.doi.org/10.1136/jitc-2020-SITC2020.0215

\section{ANTI-TUMOR ACTIVITY OF IOSH2 BY BLOCKING LILRB2 RECEPTOR SIGNALLING}

Osiris Marroquin Belaunzaran*, Anahita Rafiei, Anil Kumar, Julia Kolibaba, Lorenz Vogt, Sean Smith, Christoph Renner. ImmuOs Therapeutics AG, Schlieren, Switzerland

Background The human leukocyte immunoglobulin-like receptor family B (LILR B) acts as check point blockade of the innate immune system by inhibiting leukocyte activation through SHP phosphatase recruitment. Some of the physiological ligands include classical HLA class I molecules, including beta-2-microglobulin (B2M) free open conformers (OC). Natural HLA-OC expression is known from autoimmune disease leading to immune activation by pleiotropic effects since they bind to LILRB and KIR family members reducing Treg and MDSC numbers and increased effector T-cell and NK-cell activation, respectively. We have generated an IgG4-HLA-57 open conformer (OC) molecule (iosH2) with high affinity for LILRB molecules and demonstrate its anti-cancer activity in vitro and in vivo.

Methods iosH2 was produced by transient gene expression in $\mathrm{CHO}$ cells and purified by standard chromatography. Affinity of iosH2 binding was quantified by ELISA and SPR analysis. HLA-G mediated signaling and competition was assessed using functional cell lines. Effect of iosH2 on activation of SHP1/2 was assessed using Western Blot. Functional assays including in vitro polarization and phagocytosis potential of primary macrophages was assessed by flow cytometry in the presence of
iosH2 or isotype control. Effect of iosH2 on T cell activation was evaluated in co-cultures of cancer and $\mathrm{T}$ cells. Mouse models were used to assess in vivo activity.

Results iosH2 binds to LILRB2 with high affinity and blocks the activation of HLA-G. In addition, iosH2 blocks receptormediated activation of SHP1/2. iosH2 promotes a shift from M2 to M1 macrophages with enhanced tumor cell phagocytosis in vitro. iosH2 enhances activation and killing potential of $\mathrm{T}$ cells in cancer cells and $\mathrm{T}$ cells co-culture assay. iosH2 exerts therapeutic efficacy in mouse transgenic (melanoma) and different syngeneic tumor models (e.g. pancreatic, colon and breast cancer) as monotherapy. Moreover, it acts synergistically in vivo with PD1 blocking antibodies achieving longterm tumor control. Ex vivo tumor sample analysis demonstrates a significant reduction of MDSC and Tregs and a shift towards an activated inflammatory M1 macrophage phenotype. Loss of MDSC functionality was paralleled by enhanced CD8 $+\mathrm{T}$ cell expansion and activity.

Conclusions iosH2 binds to LILRB2 with high affinity, restores immune cell function in vitro and demonstrates anti-tumor activity in different in vivo mouse models. In addition, it acts synergistically in vivo with PD1. iosH2 is a first-in-class OC therapeutic with robust anti-tumor activity by promoting key components of the innate immune system. Clinical development is under way and phase I trial in preparation.

http://dx.doi.org/10.1136/jitc-2020-SITC2020.0216

\section{EVALUATING BIOMARKERS OF JTX-8064 (ANTI-LILRB2/ ILT4 MONOCLONAL ANTIBODY) IN AN EX VIVO HUMAN TUMOR HISTOCULTURE SYSTEM TO INFORM CLINICAL DEVELOPMENT}

Yasmin Hashambhoy-Ramsay*, Vikki Spaulding, Michelle Priess, Kristin O'Malley, Monica Gostissa, Edward Stack, Jeff Smith, Margaret Willer, Ben Umiker, Donald Shaffer. Jounce Therapeutics, Cambridge, MA, USA

Background Leukocyte immunoglobulin-like receptor B2 (LILRB2; ILT4) is an immunoinhibitory protein expressed on the surface of myeloid cells that has been increasingly recognized as a therapeutic target of interest in immuno-oncology (IO). Upon binding its ligands, MHC I molecules (e.g. HLAG/HLA-A), LILRB2 inhibits myeloid cell activation and promotes an M2-like (anti-inflammatory) state. LILRB2 was the first target prioritized from a macrophage discovery effort leading to the development of JTX-8064, a humanized monoclonal antibody that specifically binds to and antagonizes LILRB2. JTX-8064 has been shown to induce an M1-like (pro-inflammatory; anti-tumor) functional state in macrophages. Rodents do not express LILRB proteins limiting their usefulness as a model for preclinical study of JTX-8064. To overcome this limitation, we conducted an ex vivo human tumor histoculture study to assess the pharmacodynamic effects of LILRB2 antagonism. Protein and/or gene expression analysis of matched tumor samples enabled the discovery of predictive biomarkers associated with the induction of specific pharmacodynamic signatures in ex vivo-cultured human tumors in response to JTX-8064. Finally, tumor types were identified that had a high prevalence of these predictive biomarkers suggesting they may be priority indications for JTX8064 therapy.

Methods More than 100 fresh treatment-naïve human tumor samples obtained post-surgery from kidney, lung, and head and neck cancer were treated with JTX-8064 or isotype 